\title{
СТАТЬИ
}

УДК 528.11:528.06:519.65:519.2

ПОИСК ОПТИМАЛЬНОГО СПОСОБА УРАВНИВАНИЯ РЕЗУЛЬТАТОВ ПОВТОРНОГО НИВЕЛИРОВАНИЯ, ВЫПОЛНЯЕМОГО НА ГЕОДИНАМИЧЕСКИХ ПОЛИГОНАХ

Волков В.И., Волков Н.В., Волкова Т.Н.

ФГБОУ ВО «Санкт-Петербургский государственный архитектурно-строительный университет», Санкт-Петербург, е-mail: volkov.nikita@yahoo.com

В рамках научной статьи представлена реализация способа уравнивания результатов повторного высокоточного нивелирования, отличная от традиционных подходов, применяемых в геодезии. Описанный в статье метод основан на использовании, в дополнение к классическим методам математической обработки геодезических измерений, метода наименьших модулей. Выбор метода наименьших модулей в качестве инструмента для уравнивания результатов геодезических измерений обусловлен исключением влияния грубых ошибок на интерпретацию результатов наблюдений. Для решения регистрационных задач применяют метод максимального правдоподобия, однако для нормального распределения помех, метод максимального правдоподобия совпадает с методом наименьших квадратов. Теорема Гаусса - Маркова, в основе которой лежит метод наименьших квадратов, утверждает, что оценка искомых неизвестных в методе наименьших квадратов обладает наименьшей среди всех линейных несмещенных оценок матрицей ковариаций независимо от закона распределения помехи. Однако для других (отличных от нормального) законов распределения оценка методом наименьших квадратов не является эффективной. Следовательно, метод наименьших квадратов является асимптотически эффективным лишь в случае нормального закона распределения помехи, когда полученные оценки являются оценками максимального правдоподобия. «Страховка» от влияния грубых ошибок является чрезвычайно актуальным результатом исследования, позволяющим повысить качество, надежность и репрезентативность результатов сложно организуемого повторного высокоточного нивелирования. Приведенный в статье метод может быть адаптирован и к другим геодезическим наблюдениям. В статье представлены результаты и обоснование применения метода в рамках обработки результатов повторного высокоточного нивелирования на геодинамическом полигоне. Авторами показана более высокая эффективность и научная обоснованность использования метода наименьших модулей в соответствии с описанной в статье методикой по отношению к традиционно используемым в геодезии подходам к математической обработке результатов измерений.

Ключевые слова: метод наименьших модулей, уравнивание высокоточных геодезических измерений, повышение качества и репрезентативности результатов геодезических измерений

\section{SEARCH FOR THE OPTIMAL WAY OF MATHEMATICAL COMPENSATION THE RESULTS OF RE-LEVELING PERFORMED ON GEODYNAMIC POLYGONS}

Volkov V.I., Volkov N.V., Volkova T.N.

Saint-Petersburg State University of Architecture and Civil Engineering, Saint Petersburg, e-mail: volkov.nikita@yahoo.com

Within the framework of the scientific article, the implementation of a method for equalizing the results of repeated high-precision leveling, different from traditional approaches used in geodesy, is presented. The described method is based on the use of the method of least modules. This method can be used in addition to the classical methods of mathematical compensation of geodetic measurements. The choice of the method as a tool for adjusting the results of geodetic measurements is made for the elimination of the influence of gross errors on the interpretation of the results of observations. To solve registration problems, the maximum likelihood method is used, but for normal distributed interference, the maximum likelihood method coincides with the least squares method. The GaussMarkov theorem, which is based on the least squares method, states that the estimation of the desired unknowns in the least squares method has the smallest covariance matrix among all linear unbiased estimates, regardless of the interference distribution law. However, for other (non-normal) distribution laws, the least squares estimation is not effective. Consequently, the least squares method is asymptotically effective only in the case of the normal law of the interference distribution, when the estimates obtained are maximum likelihood estimates. «Insurance» against the influence of gross errors is an extremely relevant research result, which makes it possible to improve the quality, reliability and representativeness of the results of complicated repeated high-precision leveling. The method presented in the article can be adapted to other geodetic observations. The article presents the results and justification of the application of the method in the framework of processing the results of repeated high precision leveling at the geodynamic test site. The authors have shown a higher efficiency and scientific validity of using the method of least modules in accordance with the method described in the article in relation to the approaches traditionally used in geodesy to the mathematical processing of measurement results.

Keywords: method of least modules, adjustment of high-precision geodetic measurements, improving the quality and representativeness of the results of geodetic measurements

Решение многих практических и научно-технических задач, связанных с изучением современных движений земной коры и поверхности, оценкой техногенных де- формационных процессов и устойчивостью прецизионных сооружений, основано на использовании значимых и репрезентативных результатов повторных геодезических из- 
мерений. Успешное решение таких задач на современном этапе осложняется наличием в результатах повторных высокоточных нивелирований грубых погрешностей, которые часто соизмеримы с определяемыми значениями величин смещений и их скоростей. Особо следует отметить, что грубые погрешности, присутствующие в результатах повторного нивелирования, не только резко снижают эффективность их использования при мониторинге деформационных процессов, но и способствуют принятию ошибочных решений по управлению контролируемых негативных последствий, обусловленных развитием этих процессов. К причинам возникновения в результатах повторного высокоточного нивелирования следует отнести быстроизменяющиеся, нерегулярные изменения внешних условий, в которых выполняется нивелирование, нарушения методики нивелирования исполнителями и допущенные ими ошибки.

Выполненные исследования на геодинамических полигонах разрабатываемых месторождений показали, что результаты повторного нивелирования в соответствии с соответствующими нормативными документами и инструкциями отягощены в суровых климатических условиях Крайнего Севера по объективным и субъективным причинам грубыми погрешностями, обусловленными следующими основными факторами:

- сдвижениями переходных точек при различных затратах времени на прокладку прямых и обратных нивелирных ходов на фоне развития гидротермических движений земной поверхности и морозного пучения высокодисперсных грунтов;

- смещения штативов на нивелирных станциях;

случайными наклонами нивелирных реек;

- нивелирной рефракцией, порождаемой неустойчивой температурной стратификацией;

- ошибочным выбором места постановки нивелирной рейки при использовании в качестве нивелирного пункта скважин нефтегазовых промыслов или конструктивных элементов инженерных объектов;

- различия в освещении нивелирных реек и температурных деформациях разных частей нивелирных реек.

Традиционно влияние таких грубых ошибок на результаты повторного нивелирования ослаблялось на основе анализа этих результатов исполнителем в соответ- ствии с традиционными рекомендациями, приведенными в «инструкции по нивелированию I, II, III и IV классов».

На современном этапе применения автоматизированных систем геодезических измерений и математической обработки их результатов проблема оценки влияния и исключения грубых погрешностей нивелирования обострилась. Так при выполнении измерений электронными нивелирами к традиционным грубым ошибкам добавляются грубые ошибки наведения на нивелирную рейку, фиксации отсчетов по нивелирной рейке, искаженная нумерация мест постановки нивелирных реек и другие [1].

Полученная информация с помощью электронного нивелира, отягощенная грубыми погрешностями, предварительно не обрабатываемая и не анализируемая исполнителем, поступает в массив данных, подтверждающийся математической обработкой, основанной на классических способах уравнивания методом наименьших квадратов. В традиционной теории математической обработки геодезических измерений, в том числе результатов повторного нивелирования, проблеме установления наличия грубых ошибок и их исключения отводилась незначительная роль [1-3]. Поэтому необходимо дальнейшее совершенствование в геодезическом производстве процедуры поиска и исключения грубых ошибок из результатов геодезических измерений и, в частности, повторного высокоточного нивелирования. Одним из прогрессивных путей решения такой проблемы является поиск и исключение грубых ошибок в процессе уравнительных вычислений.

Целью исследования является внедрение нетрадиционного подхода к уравниванию результатов высокоточных геодезических измерений с позиций метода наименьших модулей (МНМ) [4, 5]. В рамках решаемой задачи требуется определить $k$ значений неизвестных величин из $n(n>k)$ линейных уравнений:

$$
\left(a^{i}, x\right)=b_{i}, i=1, \ldots, n ; x \in R_{k} .
$$

Метод позволяет исключить влияние грубой ошибки в измерениях на результат уравнивания, что позволит увеличить надежность и репрезентативность интерпретации результатов измерений.

\section{Материалы и методы исследования}

Уравнивание выполняется традиционно по методу наименьших квадратов (МНК), согласно которому измеренные величины 
получают поправки $v_{i}$, удовлетворяющие условию $\left[p v^{2}\right]=\min$, где $p_{i}-$ вес результата измерений. При этом применяют параметрический и коррелатный способы уравнивания. Коррелатный способ предусматривает составление $r(r=n-k)$ условных уравнений на $n$ неизвестных истинных значений измеренных величин. То есть составляются уравнения не на $k(k>n)$ неизвестных параметров, а на $n$ неизвестных истинных значений измеренных величин, представляющих собой собственно линейные комбинации $k$ неизвестных параметров.

Параметрический способ базируется на системе уравнений $n$ поправок. Основные уравнения коррелатного способа уравнивания можно представить как $(n-2)$ линейных комбинаций из $n$ уравнений поправок параметрического способа. Таким образом, коррелатный способ уравнивания, так же как и параметрический, сводится к нахождению $k$ неизвестных параметров.

Различие параметрического и коррелатного способов уравнивания состоит в том, что в параметрическом способе определяются $k$ неизвестных значений из $n$ уравнений $(n>k)$, а в коррелатном эти же $k$ неизвестных параметров определяются из $r=n-k$ уравнений, рассматриваемых как условные уравнения при минимизации некоторой функции от $n$ переменных, что делает ее функцией от $k$ неизвестных. Следовательно, оба способа уравнивания являются эквивалентными и сводятся к поиску $k$ неизвестных из $n$ при $(n>k)$ соотношений, что и составляет задачу уравнивания.

\section{Результаты исследования и их обсуждение}

Система уравнений поправок (1) является переопределенной и чаще всего не имеет решения (традиционно), поэтому ставится задача минимизации какой-либо формы от невязки (несоответствие измеренных величин известному математическому условию).

При параметрическом способе уравнивания поправки $v_{i}$ представлены как реализация помех геодезических измерений в их результатах. В соответствии помех при измерениях $n-k$ уравнения поправок системы (1) являются линейными комбинациями из $k$ независимых уравнений этой же системы.

Эту же задачу можно свести к задаче линейной регрессии типа

$$
y_{i}=\left(u^{i}, x^{*}\right)+\xi_{i}, i=1, \ldots, n ; x^{*} \in R_{k},
$$

где по $y_{i}$ (искаженные помехой $\mathrm{x}_{i}$ ) и известным входным величинам $u^{i}$ оценивается вектор $x^{*}$. Как известно [6], для решения регистрационных задач применяют метод максимального правдоподобия, здесь укажем лишь, что для нормального распределения помех метод максимального правдоподобия совпадает с методом наименьших квадратов. Известная [7] теорема Гаусса Маркова, в основе которой лежит метод наименьших квадратов, утверждает, что оценка искомых неизвестных МНК обладает наименьшей среди всех линейных несмещенных оценок матрицей ковариаций независимо от закона распределения помехи. Однако для других (отличных от нормального) законов распределения оценка МНК не является эффективной. Следовательно, МНК является асимптотически эффективным лишь в случае нормального закона распределения помехи, когда оценки МНК являются оценками максимального правдоподобия.

Согласно [7], оценки максимального правдоподобия, как и МНК при нормальном распределении помехи, не являются устойчивыми к отклонениям закона распределения помехи от предполагаемого.

Рассмотрим последние утверждения на примере. Оценкой максимального правдоподобия скалярной величины $x^{0}$ по $m$ измерениям $z_{i}=x^{0}+E_{i}$, где $E_{i}-$ независимые случайные величины, нормально распределенные со средним 0 и дисперсией 1 , является среднее арифметическое $x_{m}^{0}=\frac{\sum_{i=1}^{m} z_{i}}{m}$.

Представленная оценка имеет наименьший второй момент среди любых несмещенных оценок для случая нормального распределения помех. Пусть одно измерение $z_{i}$ из $m$ измерений $z_{m}$ распределено иначе. Тогда и у оценки $x_{m}^{0}$ дисперсия будет значительной по величине. Такие ситуации сопровождает высокоточное повторное нивелирование, выполненное в циклах наблюдений на геодинамических полигонах (отказ нивелира, смещение костыля и нивелирного знака, ошибочное наблюдение нивелирной рейки и т.д.). Такие события встречаются при строгом соблюдении программы нивелирования редко, но оказывают решающее влияние на оценку $x_{m}$.

Швейцарский статистик Хубер [8] предложил путь исключения таких осложнений. Считая, что истинная плотность помехи $p$ неизвестна (известным является лишь класс, которому она принадлежит), Хубер указал оценки, работоспособные для всех $p$ из этого класса. При естественных предпо- 
ложениях такие оценки являются асимптотически мини-максными на класс, то есть в известном смысле [6] оптимальными. Приведем Хуберовские оценки для двух наиболее важных классов. Если $p_{m}-$ класс всех нивелирных распределений, то

$$
x_{m}^{u}=\arg \min f_{m}(x),
$$

где

$$
f_{m}(x)=\sum_{i=1}^{m}\left(y_{i}-\left(u_{i}, x\right)\right)
$$

То есть поскольку о распределении ничего не известно, то следует применять метод наименьших модулей. Если $p$ - класс «приближенно нормальных» распределений, то

$$
\begin{gathered}
x_{m}^{u}=\arg \min f_{m}(x)=\sum_{i=1}^{m} F\left(y_{i}-\left(u_{i}, x\right)\right), \\
F(z)=\left\{\begin{array}{c}
\frac{z^{2}}{r},|z| \leq d \\
|z| \cdot d-\frac{d^{2}}{r},|z|>d
\end{array}\right.
\end{gathered}
$$

Параметр $d$ в математических выражениях (3) зависит от «уровня загрязнения» основного (нормального распределения). Следовательно, если распределение близко к нормальному, то необходимо пользоваться «промежуточным» между МНК и МНМ методом. Следовательно, использование МНМ предоставляет возможности «застраховаться» от влияния грубой ошибки измерений, когда о распределении помехи ничего не известно. Следует учитывать, что МНК в этой ситуации может дать оценку неизвестного вектора параметров, непригодную для научно-практического использования в геодинамической и геомеханической интерпретации результатов повторного нивелирования [9].

Рассмотрим результаты уравнивания элементов нивелирной сети, представленной на рисунке.

При параметрическом способе уравнивания система уравнений поправок (1) имеет следующий вид

$$
\begin{gathered}
v_{i}=a_{i} \delta x_{1}+b_{i} \delta x_{2}+\ldots+g_{i} \delta x_{k}+l_{i}, \\
l_{i}=f_{i}\left(x_{1}^{0}, x_{2}^{0}, \ldots, x_{k}^{0}\right)-y_{i},
\end{gathered}
$$

где $x_{i}^{0}$ - близкие (приближенные) к точным значениям измеряемых параметров.

В таблице представлены значения поправок $\mathrm{d} x$, полученных по рекуррентным схемам МНК и МНМ при трех рядах значений невязок $l_{k j}(k=1, \ldots, 6 ; j=1,2,3)$ и соответствующих им дисперсиям невязок $d_{i}$. Представленные результаты показывают, что при уменьшении «дисперсии невязок» значения поправок $d x_{1}, d x_{2}, d x_{3}$, полученных по рекуррентным схемам МНК и МНМ, сближаются, а при увеличении «дисперсии невязок» в значениях поправок наблюдаются значительные расхождения. Так как о распределении погрешностей в рассматриваемом примере ничего не известно, то для уравнивания, с позиций научной корректности и эффективности результатов уравнивания, целесообразно применить рекуррентный метод наименьших модулей [9, 10$]$.

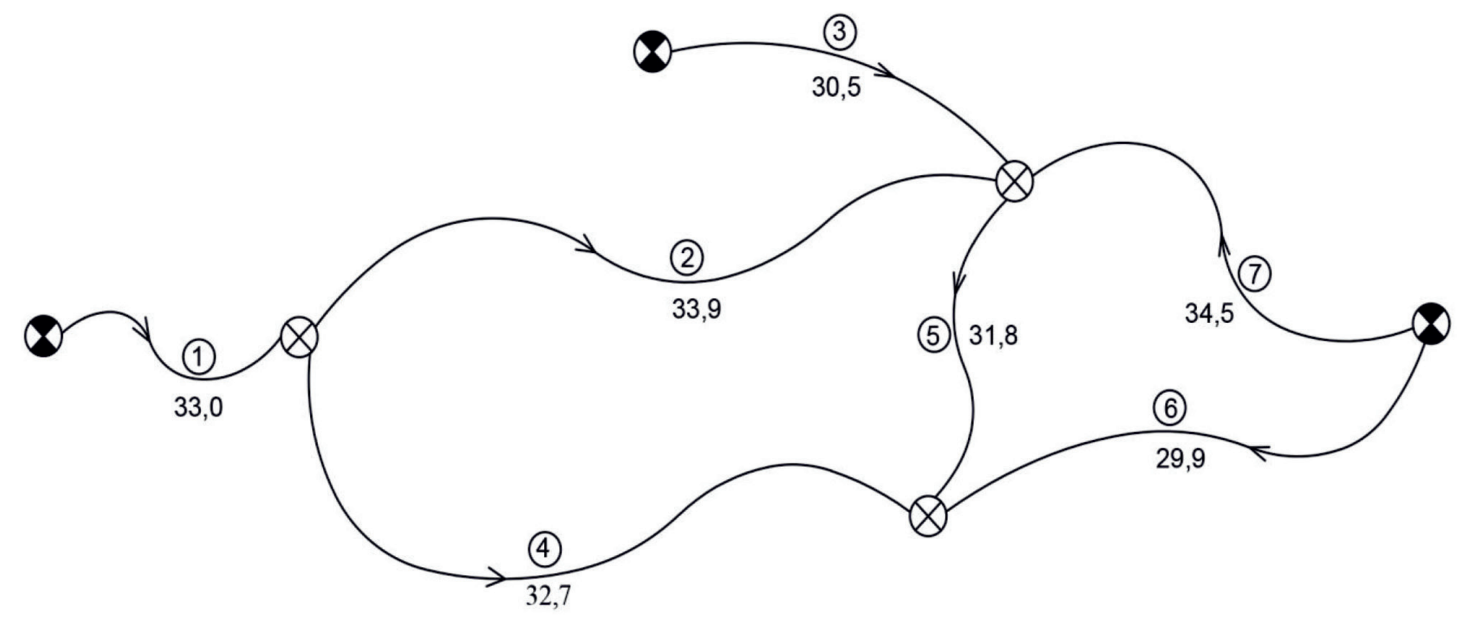

Схема сети нивелирных ходов 
Результаты уравнивания

\begin{tabular}{|l|c|c|}
\hline \multicolumn{1}{|c|}{ Ряд невязок $l_{i}$, мм } & \multicolumn{2}{|c|}{ Метод уравнивания и значения поправок $\delta_{x}$} \\
\cline { 2 - 3 } & МНК & МНМ \\
\hline Дисперсии невязок $\delta_{i}$, мм & $\delta \mathrm{x}_{1}, \delta \mathrm{x}_{2}, \delta \mathrm{x}_{3}$ & $\delta \mathrm{x}_{1}, \delta \mathrm{x}_{2}, \delta \mathrm{x}_{3}$ \\
\hline 0,$0 ; 1,7 ; 0,0 ; 8,5 ; 4,9 ; 0,0 ;-0,9$ & $-2,57-0,823,30$ & $-2,00-0,304,50$ \\
12,37 & & \\
Критерий МНК $=25,56$ & $-1,48-1,30-0,62$ & \\
Критерий МНМ $=9,40$ & & $-1,13-1,33-0,72$ \\
\hline$-1,0 ; 1,2 ;-1,3 ; 1,4 ; 1,1 ;-1,2 ; 1,5$ & & \\
1,41 & $-2,56-0,823,31$ & \\
Критерий МНК $=1,81$ & & \\
Критерий МНМ $=2,79$ & & \\
\hline 0,$0 ; 1,65 ; 0,0 ; 8,5 ; 4,8 ; 0,0 ; 0,9$ & & \\
12,35 & & \\
Критерий МНК $=25,56$ & & \\
Критерий МНМ $=9,42$ & & \\
\hline
\end{tabular}

Справедливость данной рекомендации по выбору метода для уравнивания результатов нивелирования также подтверждается следующими обстоятельствами. В рассматриваемой нивелирной сети геодинамического полигона при равных длинах ходов и периметров полигонов нивелирования получены значительные расхождения в невязках от 0,9 до 8,5 мм, что позволяет предположить наличие отклоненной от нормального закона распределения ошибок нивелирования. Это частично подтверждается данными уравнивания, полученными по МНК и МНМ.

\section{Заключение}

Таким образом, применение традиционно используемых для решения подобного рода задач методов, относящихся к математической обработке результатов повторных высокоточных геодезических наблюдений, в основе которых лежит метод наименьших квадратов, не является полностью обоснованным и единственным безальтернативным подходом. Приведенные результаты и требования нормативных материалов, накладываемые на методику проведения повторного высокоточного нивелирования, показывают, что применение метода наименьших модулей, в дополнение к методу наименьших квадратов, позволяет повысить качество, репрезентативность и релевантность результатов повторного высокоточного нивелирования, за счет снижения влияния систематических ошибок повторного высокоточного нивелирования и позволяя исключить их или учесть на стадии обработки его результатов.

\section{Список литературы / References}

1. Герасименко М.Д., Каморный В.М. О методах поиска и отбраковки грубых ошибок геодезических измерений // Вестник инженерной школы ДВФУ. 2018. № 3. С. 128-133.

Gerasimenko M.D., Kamornyy V.M. On methods of searching and rejecting gross errors of geodetic measurements // Vestnik inzhenernoj shkoly DVFU. 2018. No. 3. P. 128-133 (in Russian).

2. Зубарев А.Э., Федосеев Ю.В. Особенности методики математической обработки результатов высокоточного нивелирования с использованием электронных нивелиров // Автоматизированные технологии изысканий и проектирования. 2011. № 4. С. 76-81.

Zubarev A.E., Fedossev Yu.V. Features of the methodology for mathematical processing of the results of high-precision leveling using electronic levels // Avtomatizirovannye tekhnologii izyskanij i proektirovaniya. 2011. No. 4. P. 76-81 (in Russian).

3. Беликов А.Б., Симонян В.В. Математическая обработка результатов геодезических измерений. НИС МГСУ. 2016. 432 c.

Belikov A.B., Simonyan V.V. Mathematical processing of the results of geodetic measurements. NIS MGSU. 2016. 432 p. (in Russian).

4. ГОСТ 20522-2012. Грунты. Методы статистической обработки результатов испытаний: национальный стандарт Российской Федерации: издание официальное: утвержден и введен в действие Приказом Федерального агентства по техническому регулированию и метрологии от 29 октября 2012 г. № 597-ст: дата введения 2013 07-01. М.: Стандартинформ, 2012. $16 \mathrm{c}$.

GOST 20522-2012. Soils. Methods of statistical processing of test results: national standard of the Russian Federation: official edition: approved and put into effect by the Order of the Federal Agency for Technical Regulation and Metrology dated October 29, 2012. No. 597-st: date of introduction 2013-07-01. Standartinform, 2012. $16 \mathrm{p}$.

5. Пантелеев А.В., Летова Т.А. Методы оптимизации в примерах и задачах. М.: Высшая школа, 2015. 544 с.

Panteleev A.V., Letova T.A. Optimization methods in examples and problems. M.: Vysshaya shkola, 2015.544 p.

6. Поляк Б.Т. Введение в оптимизацию. М.: Наука, 1983. $384 \mathrm{c}$ $384 \mathrm{p}$.

Polyak B.T. Introduction to optimization. M.: Nauka, 1983.

7. Большаков В.Д., Маркузе Ю.И., Голубев В.В. Уравнивание геодезических построений. М.: Недра, 1989. 413 с.

Bol'shakov V.D., Markuze Yu.I., Golubev V.V. Adjustment of geodetic constructions. M.: Nedra, 1989. 413 p. 
8. Peter Liver: Huber Eugen.In: Neue Deutshe Biographie (NDB), Band 9, Duncker \& Humblot, Berlin, 1977. 690 p.

9. Волков В.И., Митягин С.Д., Волкова Т.Н. Новый подход к математической обработке результатов повторных геодезических наблюдений, используемых в архитектурностроительной практике // Вестник гражданских инженеров. 2015. № 6 (53). C. 216-221.

Volkov V.I., Volkova T.N., Mityagin S.D. A new approach to the mathematical processing of the results of repeated geo- detic observations used in architectural and construction practice // Vestnik grazhdanskih inzhenerov. 2015. № 6 (53). P. $216-$ 221 (in Russian).

10. Герасименко М.Д., Каморный В.М. Уравнивание повторных геодезических измерений при наличии систематических ошибок // Геодезия и картография. 2014. № 9. С. 6-8.

Gerasimenko M.D., Kamornyy V.M. Adjustment of repeated geodetic measurements in the presence of systematic errors // Geodeziya i kartografiya. 2014. № 9. P. 6-8 (in Russian). 